\title{
ROAD NETWORK ANALYSIS FOR RISK AND RESILIENCE ASSESSMENT FRAMEWORK OF ROAD INFRASTRUCTURE SYSTEMS
}

\author{
SILVIA IENTILE ${ }^{1}$, FRANZSIKA SCHMIDT ${ }^{1}$, CHRISTOPHE CHEVALIER $^{2}$, ANDRE ORCESI $^{1}$, \\ LUCAS ADELAIDE $^{1} \&$ BOUMEDIENE NEDJAR $^{1}$ \\ ${ }^{1}$ MAST-EMGCU, IFSTTAR, University Gustave Eiffel, France \\ ${ }^{2}$ GERS-SRO, IFSTTAR, University Gustave Eiffel, France
}

\begin{abstract}
Maintenance and protection of road infrastructures (RI) against multi-hazard events requires the use of risk assessment to identify threats, to assess vulnerabilities and to evaluate the impact on infrastructure systems according to the probability of occurrence of such threats. Dealing with a complex network system, such as RI, demands the improvement of the risk assessment approach by resorting to the concept of resilient infrastructure, capable of dealing with scenarios linked to disastrous events with the aim of minimizing service interruptions and quickly recovering. In this paper, the network analysis of an RI case study is addressed to obtain a qualitative measure of resilience related to travel patterns of road network. The topology of RI system, a pattern of connections according to graph theory, between components interacting jointly to provide the required functionalities, is supplied by road network data from OpenStreetMap. Then a probabilistic system model is used to define a non-degradated condition and the scenarios of failure events that can affect the network system. For each condition, the evaluation of the related shortest paths, as a measure of the network system performance, provides the qualitative resilience measurement identifying performance criteria or levels of service requirements. The proposed assessment through transport network measures can find application in risk assessment and management by RI owners and managers.
\end{abstract}

Keywords: risk analysis, resilience, critical infrastructures, complex network, road infrastructure, vulnerability assessment.

\section{INTRODUCTION}

Road infrastructure systems have been increasingly exposed to natural hazards due to climate change in recent years [1]. There is the need to address the vulnerability of infrastructure to raise its coping and adapting capacity to the impact of the multi-hazard events such as earthquake, flooding, landslides, etc.. Reducing the vulnerability of road RI and thus improving the resilience of the system leads to a mitigation of the social and economic consequences, which are often a significant cost for transport agencies and operators [2]. Therefore the maintenance and protection of RI requires effective risk assessment and mitigation methodologies.

A large number of risk assessment methodologies exist for infrastructures including RI, in particular those defined as critical. The domain of applicability, i.e. the asset level, the infrastructure/system level or the system of a system level (as part of a larger system), determines the users (policy makers, authorities, operators and asset managers) and the results of method. Existing methodologies [3] consider the infrastructure starting from the concept of complex system until the system of system in order to obtain a risk assessment taking into account technical, organizational, social and economic aspects. This crosssectorial approach opens to new methods for the assessment of RI introducing the concept of infrastructure resilience.

The analysis of complex networks, in the case of RI networks, mostly covers research fields that concern geography and regional sciences addressing accessibility issues [4]. In 
this paper, the aim is to use network analysis to assess the performance of a road infrastructure network in risk and resilience assessment framework.

\section{RISK AND RESILIENCE ASSESSMENT}

Risk assessment framework usually follows a linear approach consisting in the identification and classification of threats, the identification of vulnerabilities and the evaluation of the impact [5]. In case of complex network system such as RI, it is sensibly improved when considering the additional elements of interdependencies and interconnections [6], [7]. For this purpose, the definition of resilience concept is necessary, understood as the capacity of a system to plan for, recover from and adapt to adverse events overtime, as defined by USA National Academy of Science. Then, the risk analysis estimates the entity functionality loss of a system due to hazard events, while the resilience represents the related absorption and recovery curves over time (Fig. 1).

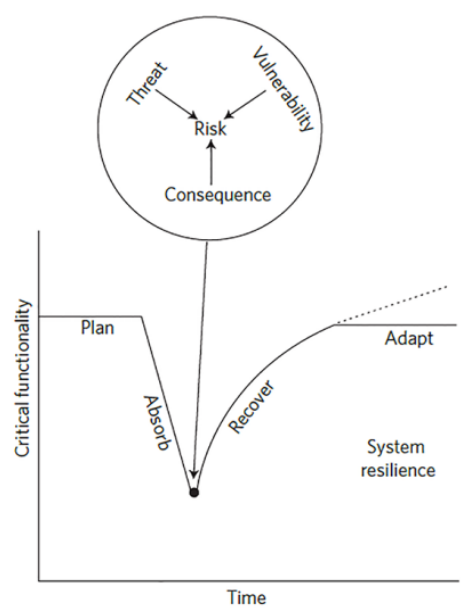

Figure 1: Resilience management framework [6].

A definition of critical infrastructure resilience is given by RESILENS Project: "a transformative, cyclical process that builds capacities in technical, social and organisational resources for critical system function, so as to mitigate the impacts of disruptive events and long-term incremental changes, thus guaranteeing the continued provision of its basic functions. Critical infrastructure resilience is based upon new forms of risk management, adaptability and the assessment of potential trade-offs between parts of a system" [8].

Therefore, a resilient infrastructure is capable of dealing with scenarios linked to disastrous events with the aim of minimizing service interruptions and quickly recovering.

The following sections propose a complex network analysis that takes into account the characteristics of the risk and resilience assessment framework of a RI network. Once the natural hazard to which a road network is exposed has been identified, the vulnerability is constituted by the failure of its connections, i.e. the roads whose consequences can be quantified. The loss of functionality due to a disruptive event can affect the normal use of the network by the community. The risk assessment of the system through the evaluation of the functional importance of the connections also allows measuring its resilience considering the alternative solutions of the network structure [9]. 


\section{NETWORK ANALYSIS FOR ROAD INFRASTRUCTURE}

\subsection{Road transportation network and road vulnerability}

The study of transport networks, especially in the social sciences, has progressed thanks to the contribution of geographic information systems (GIS) and spatial economics. Thus, from the limited study of small networks analysis, since the 1990s the analyses of structures and dynamics move to large-scale networks of all kinds, including transport networks.

The classical graph theory is integrated with complex network methods used by social scientists such as geographers, economists and regional scientists, who are the majority of researchers in the field of transport networks.

To characterize transport networks, one can refer to the static dimension, i.e. the structure of a network (topology, morphology, geometry and spatial structure), and to the dynamics that investigate the evolution of the network over time and properties underlying such evolution [10]. The structure of a transport network is a very important aspect since it belongs to the category of spatial networks characterized by the transportation mode. In the case of road or rail infrastructures, one can refer with a good approximation to planar networks. It is defined by a two-dimensional Euclidean space whose edges are real physical connections. By simplifying its real structure in a graph, as shown in Fig. 2, i.e. groups of nodes (vertexes) connected by some links (edges), a wide set of concepts and measures from graph theory can be applied to RI-networks [11].

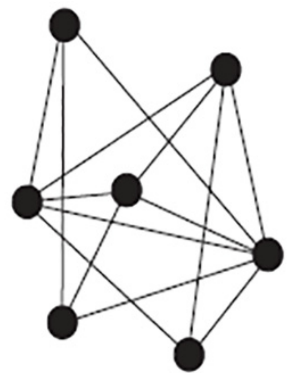

(a)

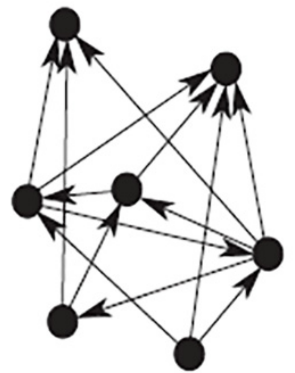

(b)

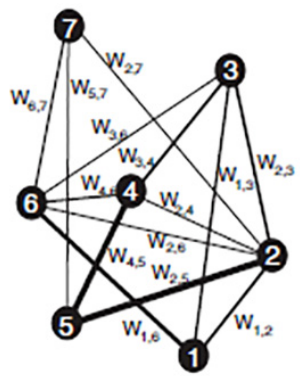

(c)

Figure 2: Graphical representation of complex networks structures. (a) Undirected graph; (b) Directed graph; and (c) Weighted undirected graph [11].

Some of these measures, such as network size (number of nodes and sides), network length and diameter, network continuity in terms of connected components, connectivity and nodality, are used to determine indices and qualitative descriptions of transportation network topology and its performance [12], [13]. While, referring to the calculation of the optimal route from the network through algorithms, the evaluation of the average length of the shortest path can allow measuring the efficiency of a network [14].

These methods and models for the study of the road transport networks mentioned above must be combined with the concept of road vulnerability for the protection against the risks and natural hazard they are exposed to. A reduction of capacity/operation for a road transport system can occur for various reasons, such as physical collapse or deterioration of structures or the reduction of lanes due to maintenance activities. A possible definition is given by [15] considering all relevant related concepts: "Vulnerability in the road transportation system is 
a susceptibility to incidents that can result in considerable reductions in road network serviceability". This last concept addresses the functioning of a road, considered as the supply side of a road network, so the serviceability of an edge or, at a global scale, of a road network represents the possibility to use it during a given time period. Therefore it is possible to assess the vulnerability of a road network considering the functioning or non-functioning state of its components, evaluating the consequences according to adequate service levels. Three aspects can be considered in the evaluation namely connectivity reliability, travel time reliability and capacity reliability.

If we refer to the probability of occurrence of an accident, the reduction in serviceability is determined by the potential failure of one edge, which then affects the three aspects mentioned above. The impact at the network level is assessed through indexes that measure the loss of serviceability knowing the morphology of the network [16] concerning connectivity and travel time reliability. Road network is represented by a weighted directed or multi-directed graph in which each edge has a value corresponding to the distance along the roads. A geodesic path, commonly called shortest path, is used by the classical graph theory to consider the travel time reliability. It is the time path between two vertices such that no shortest time path exists; the related shortest distance (time travel) is evaluated resorting to specific algorithms [17]. In this work, the calculation of shortest path between two points, A and B via a road network, takes into account the lengths or the travel times resorting to a specific algorithm, such the widely used Dijkstra's algorithm. It finds the shortest distance from a given source node to every other node in the considered network by recording all the shortest distance calculated to each vertex and at the end keeping the shortest distance possible by any route.

\subsection{Methodology for a road network analysis}

Initially it is necessary to obtain the structure of the road network in order to represent under a graph-structure. In addition to the number of nodes and edges, the road hierarchy according to their classification (motorway, trunk road, primary, etc.) and edges attributes (length, number of lanes, maximum speed, etc.) should be provided. The extension and specific features of a road network can be an issue in the modeling process [18], as not all roads, i.e. the edges, present in the network have a strategic role or relevant importance to the purpose of the network analysis. Therefore, a process of simplification must follow the identification of the network structure, in which the identification of node must give priority to those determined by junction, namely any connection point between different roads and by road ends.

In order to obtain the structure of the road network and thus the above-mentioned road asset data, OpenStreetMap (OSM) was used since it can be considered a globally reliable and complete source of transport infrastructure data of the world [19], [20]. OSM road asset data are retrieved and processed through OSMNx, an open-source Python package, which includes all the functions for the network analysis [21].

The vulnerability of the road network is assessed considering the travel time as a measurement of network performance. A sample of shortest paths weighted by travel time is then evaluated according to a set of $n$ randomly nodes within the network as points of origin $i$ and destination $j$. This is considered the non-degradated configuration of the road network, where the serviceability of all edges is not changed by any failure. A reference value $\alpha$ is calculated for this scenario as the sum of the travel times $t$ of the shortest paths considered, as in eqn (1) 


$$
\alpha=\sum_{i=1}^{n} \sum_{j=1}^{n} t(i, j) .
$$

Subsequently a degradated configuration is considered, in which $k$ edge/road of interest, defined as impactful edges, against which the vulnerability is to be measured, are eliminated one by one. For each of these configurations, the shortest paths and their travel times are recalculated, considering the same origin and destination nodes of the non-degradated configuration. Then for each edge is calculated $\beta_{\mathrm{k}}$ according to eqn (2)

$$
\beta_{k}=\sum_{i=1}^{n} \sum_{j=1}^{n} t(i, j) .
$$

These two measures are combined to obtain an estimate of the network vulnerability considering the failure of a specific edge, so an edge vulnerability index (EVI) is evaluated as in eqn (3)

$$
E V I_{k}=\frac{\beta_{k}-\alpha}{\alpha} .
$$

\section{RESULTS}

Using the presented methodology, a network analysis has been carried out considering a stretch of the A2 Highway, in the province of Guadalajara in Spain, that connects Madrid to Barcelona, one of the pilot study of the PANOPTIS project [22] under which this research has been carried out. The selected stretch has a strategic function in Spanish RI system, indeed it links the two largest cities of the country.

For the generation of the graph, twelve locations nearby the section of the analysed motorway stretch have been selected, as shown in Fig. 3(a). The simplification of the graph, from which the edges classified as living and residential streets have been eliminated, led to a graph of 2281 nodes and 4196 edges (Fig. 3(b)). Therefore, this constitutes the basic network structure, i.e. the non-degradated configuration of the road network. The relative performance is evaluated exclusively considering the failure of motorway edges of the network. With a random choice among all the nodes of the network, 15 sets were determined, each of them comprising of 6 nodes which are the origin and destination points whose shortest paths weighted by travel time are to be determined. For each set, $\alpha$ value is calculated and then by means of an iterative algorithm the motorway edges that are involved in the shortest paths of the non-degradated configuration are identified. Thus the latter are removed

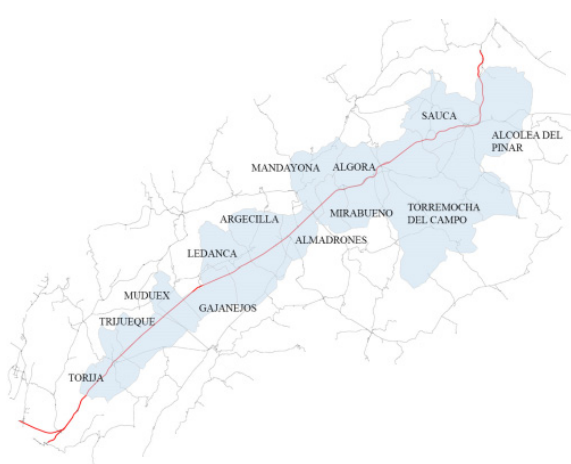

(a)

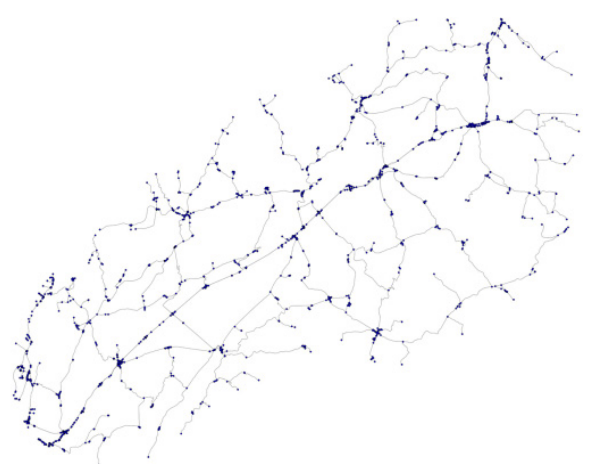

(b)

Figure 3: (a) A2 Highway highlighted in red in the network graph by OSM; and (b) Simplified network graph of the selected road network. 
one-by-one constituting several degradated configurations, for each of them the EVI value is calculated.

Table 1 shows the results for all the sets considered in the network analysis, for each set a certain number of motorway edges are involved defined as impactful. Among them, in some cases, some edges have been identified as essential. In fact, for specific nodes and related paths of the network, there are edges necessary to make that node accessible. When an essential edge is removed, the network structure is disconnected, and the paths that include the inaccessible node does no longer exist.

Table 1: Results of the network analysis for each node set.

\begin{tabular}{|c|c|c|c|}
\hline SET & Impactful edges & Essential edges & Maximum EVI \\
\hline 1 & 69 & - & $5.29 \%$ \\
\hline 2 & 138 & 4 & $10.24 \%$ \\
\hline 3 & 111 & - & $5.70 \%$ \\
\hline 4 & 140 & - & $19.37 \%$ \\
\hline 5 & 148 & 1 & $9.01 \%$ \\
\hline 6 & 108 & 4 & $15.02 \%$ \\
\hline 7 & 111 & 1 & $5.48 \%$ \\
\hline 8 & 30 & - & $12.19 \%$ \\
\hline 9 & 140 & - & $5.45 \%$ \\
\hline 10 & 85 & 3 & $8.86 \%$ \\
\hline 11 & 130 & 8 & $10.26 \%$ \\
\hline 12 & 137 & - & $6.44 \%$ \\
\hline 13 & 111 & 11 & $7.44 \%$ \\
\hline 14 & 123 & - & $9.04 \%$ \\
\hline 15 & 127 & 13 & $13.15 \%$ \\
\hline
\end{tabular}

Given the total number of motorway edges, i.e. 225 , which belong to the road network in question, almost half of the sets of the analysis involve about half of them. Therefore these sets provide a more comprehensive assessment of the motorway stretch covered by this study. The maximum EVI value identifies which and how much the failure of an edge/road mostly affects the serviceability of the road network for that particular group of origin-destination nodes. With regard to this, in case there are essential edges, it should be pointed out that the related EVIs calculated are not comparable with those obtained by all the other sets. In fact, it is not possible to obtain vulnerability indices for the essential edges, and the assessment of their influence on the network performance is limited. The network is disconnected and the global serviceability is compromised by this "local anomaly", in this case, one does not refer to a degradated configuration but to a failure configuration of the network.

Considering this, it can be noted that in Set 4 and Set 9, given the same number of impactful edges, widely different maximum EVI values are obtained. Fig. 4 shows that in the analysis of the two sets, both groups of origin-destination nodes and their shortest paths include a large majority of edges along the linear development of the motorway stretch. In Set 4 , the functionality of the road network is more affected when the edges highlighted in red fail, reaching index values between $15 \%$ and $20 \%$. 


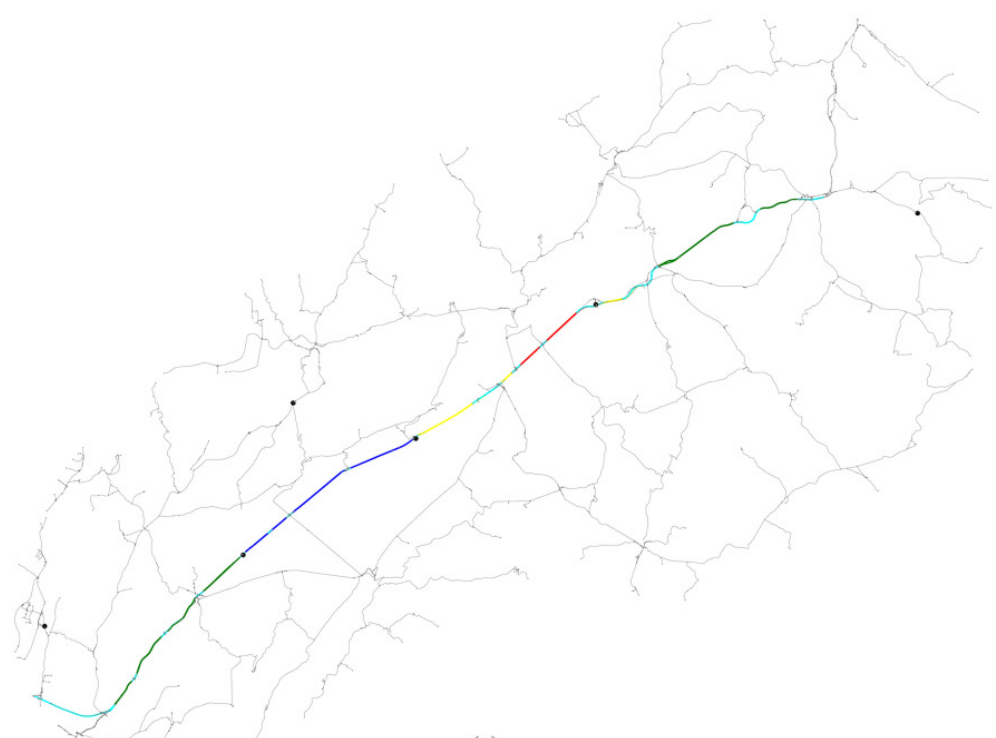

(a)

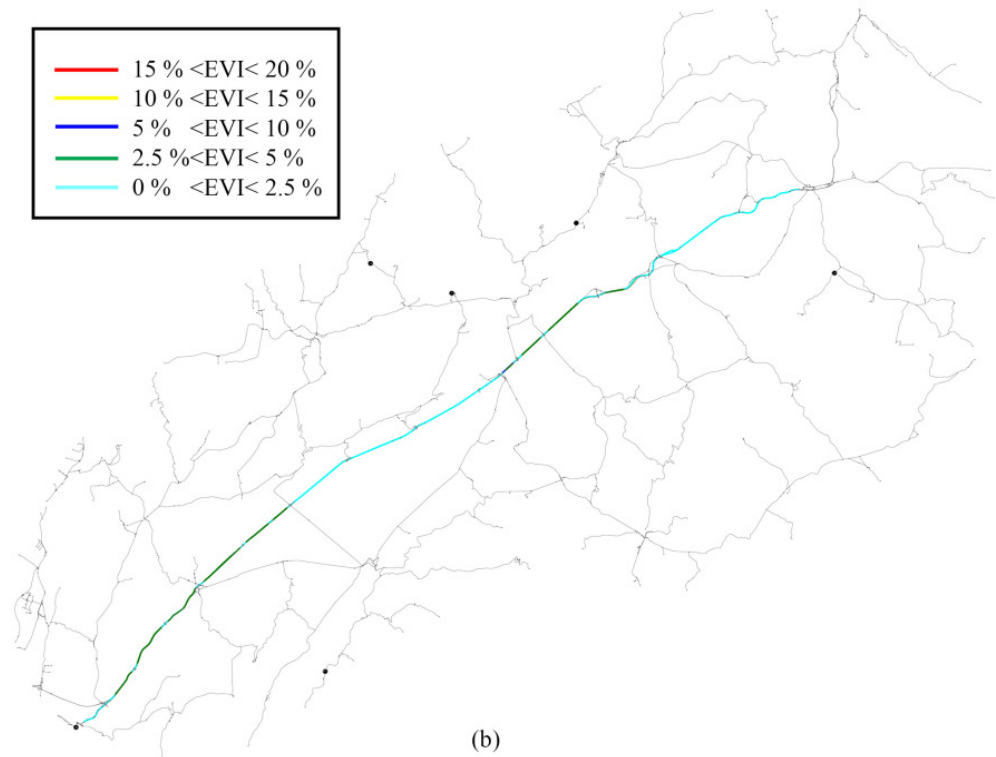

Figure 4: Graph representation of A2 Highway and EVI values in the network analysis of (a) Set 4; and (b) Set 9. Black points are the origin-destination nodes.

However, the same edges do not determine the same degree of degradation of the network; indeed in Set 9 (Fig. 4(b)) one can note that the index values are much lower than in the Set 4 (Fig. 4(a)). This is certainly determined by the fact that the indexes are calculated considering different nodes and paths for each seat. Therefore, as in this case, it can be said that the travel time of the alternative route influences the degree of impact of that edge/road on the road network. 
To confirm that the vulnerability index is proportionally correlated to the travel time of the alternative shortest path, the results Set 8 are shown in Fig. 5. The group of origindestination nodes identifies a narrower portion of the stretch under analysis, whose involved motorway edges show a degree of vulnerability similar to Set 9 in Fig. 4(b) (except for the local peak, where the maximum value of EVI is identified).

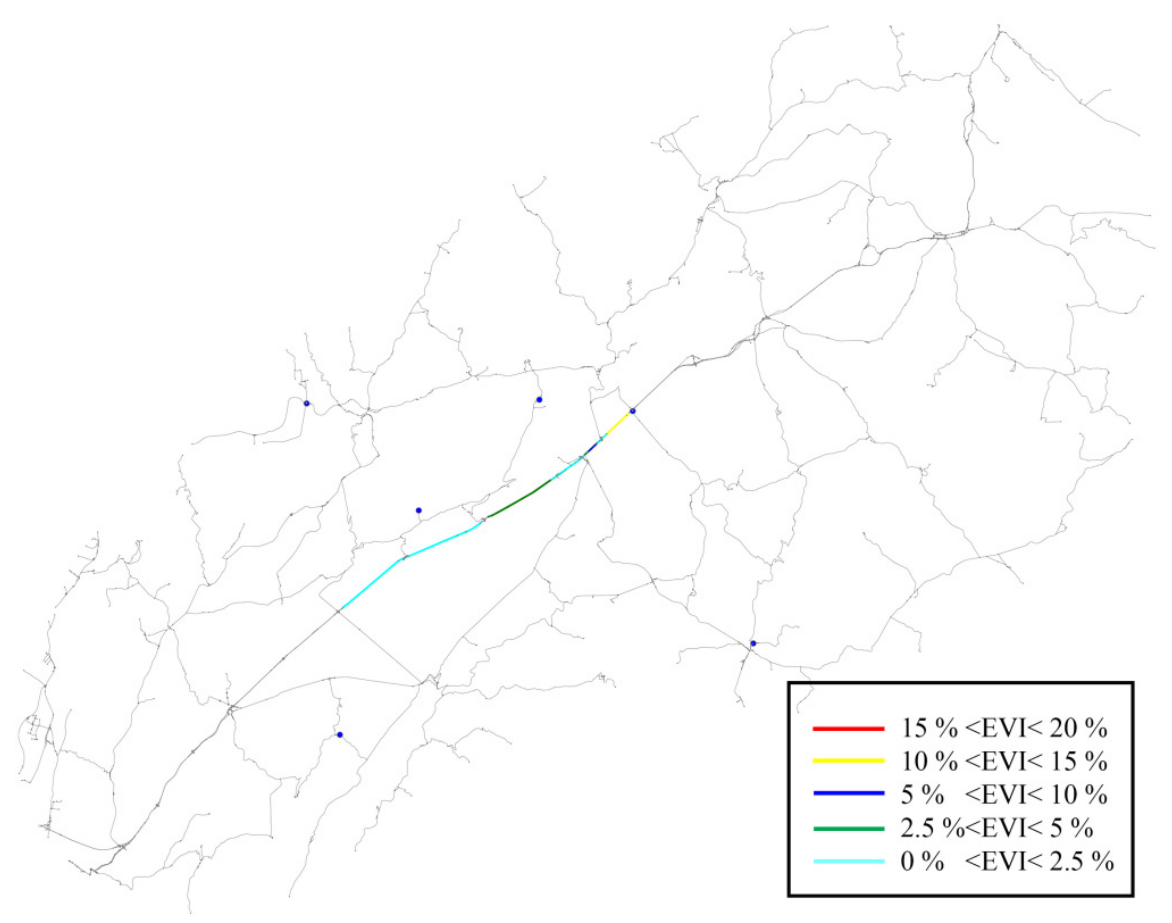

Figure 5: Graph representation of A2 Highway with EVI values in the network analysis of Set 8 .

\section{CONCLUSIONS}

The network analysis is based on the modelling of a system such as road infrastructure through the basic theory of graphs. Its use allows evaluating the impact of a road failure on the performance of the road network, determined by disruptive events, such as earthquakes, floods, structural collapses, etc.. The analysis carried out in this paper has used the network structure and a measure of optimal route, such as the shortest path, addressing the system serviceability. For this purpose, two configurations of the network are taken into account, a non-degradated in which the operation of all edges/roads is assured and a degradated one, through an iteratively process of road failure. The obtained EVI indices have measured the loss of serviceability by combining the two configurations, which then allows to quantify the road vulnerability of the network. This is linked to the measurement of the travel time reliability strongly influenced by the predisposition of the road network to supply alternative routes in case of road failure.

This measure of local vulnerability can also be interpreted as a global measure of the road network performance, affected by road failures. Then the vulnerability index provides a 
quantification of the consequent loss in network serviceability. Moreover, the existence of alternative routes, which therefore allow maintaining a degree of system functionality, represents its resilient capacity.

\section{ACKNOWLEDGEMENT}

Financial support has been provided by the Innovation and Networks Executive Agency (INEA) under the powers delegated by the European Commission through the Horizon 2020 program "PANOPTIS-development of a decision support system for increasing the resilience of transportation infrastructure based on combined use of terrestrial and airborne sensors and advanced modelling tools", grant agreement number 769129.

\section{REFERENCES}

[1] Koks, E.E. et al., A global multi-hazard risk analysis of road and railway infrastructure assets. Nature Communication, 10(2677), 2019.

[2] Nemry, F. \& Demirel, H., Impacts of climate change on transport: A focus on road and rail transport infrastructures. EUR - Scientific and Technical Research Reports, Publications Office of the European Union, 2012.

[3] Giannopoulos, G., Filippini, R. \& Schimmer, M., Risk assessment methodologies for critical infrastructure protection. Part I: A state of the art. EUR - Scientific and Technical Research Reports, Publications Office of the European Union, 2012.

[4] Kuby, M., Tierney, S., Roberts, T. \& Upchurch, C., A comparison of geographic information systems, complex networks, and other models for analyzing transportation network topologies. NASA Center for AeroSpace Information (CASI), Contractor Report No. 2005-213522, 2005.

[5] Baker, G.H., A Vulnerability assessment methodology for critical infrastructure facilities. Proc. Dep. Homel. Secur. 2005 Res. Symp., Feb., pp. 1-15, 2005.

[6] Linkov, I. et al., Changing the resilience paradigm. Nat. Clim. Chang., 4(6), pp. 407409, 2014.

[7] Klaver, M.H.A., Luiijf, H.A.M., Nieuwenhuijs, A.H., Cavenne, F., Ulisse, A. \& Bridgeman, G., European risk assessment methodology for critical infrastructures. 1st Int. Conf. Infrastruct. Syst. Serv. Build. Networks a Bright. Futur. INFRA 2008, 2008.

[8] RESILIENS consortium, Methods for Resilience Assessment, p. 138, 2016.

[9] Gleyze, J.F., La vulnérabilité structurelle des réseaux de transport dans un contexte de risques, Géographie, Université Paris-Diderot -- Paris VII, Français, 2005.

[10] Ducruet, C. \& Lugo \& I., Structure and dynamics of transportation networks: Models, methods and applications. The SAGE Handbook of Transport Studies, eds J.P. Rodrigue, T.E. Notteboom \& J. Shaw, SAGE, pp. 347-364, 2013.

[11] Boccaletti, S., Latora, V., Moreno, Y., Chavez, M. \& Hwang, D.-U., Complex networks: Structure and dynamics. Physics Reports, 424(4-5), pp. 175-308, 2006.

[12] Scott, D.W., Novak, D., Aultman-Hall, M. \& Guo, F., Network robustness index: A new method for identifying critical links and evaluating the performance of transportation networks. Centre for Spatial Analysis, Working Paper No. 9, 2005.

[13] Xie, F. \& Levinson, D., Measuring the structure of road networks. Geographical Analysis, 39, pp. 291-307, 2007.

[14] Barabási, A.L. \& Albert, R., Statistical mechanics of complex networks. Review of Modern Physics, 74, pp. 47-97, 2002.

[15] Berdica, K., An introduction to road vulnerability: What has been done is done and should be done. Transport Policy, 9, pp. 117-127, 2002. 
[16] Appert, M. \& Laurent, C., Measuring urban road network vulnerability using graph theory: The case of Montpellier's road network, 2007. https://halshs.archives-ouvertes.fr/halshs-00841520.

[17] Newman, M., Networks: An Introduction, Oxford University Press, 2010.

[18] Marshall, S., Line structure representation for road network analysis. Journal of Transport and Land Use, 9(1), pp. 29-64, 2015.

[19] Barrington-Leigh, C. \& Millard-Ball, A., The world's user-generated road map is more than $80 \%$ complete. PLoS ONE, 12(8), 2017.

[20] Meijer, J., Huijbregts, M.A.J., Schotten, K. \& Schipper, A., Global patterns of current and future road infrastructure. Environmental Research Letters, 13(6), 2018.

[21] Boeing, G., OSMnx: New methods for acquiring, constructing, analyzing, and visualizing complex street networks. Computers, Environment and Urban Systems, 65 , pp. 126-139, 2017.

[22] PANOPTIS project. http://www.panoptis.eu/. 